\title{
CDT and the big bang
}

Ambjorn, J.; Watabiki, Y.

Published in:

Acta Physica Polonica B, Proceedings Supplement

DOI:

10.5506/APhysPolBSupp.10.299

Publication date:

2017

Document version

Publisher's PDF, also known as Version of record

Document license:

CC BY

Citation for published version (APA):

Ambjorn, J., \& Watabiki, Y. (2017). CDT and the big bang. Acta Physica Polonica B, Proceedings Supplement, 10(2), 299-303. https://doi.org/10.5506/APhysPolBSupp.10.299 


\title{
CDT AND THE BIG BANG*
}

\author{
J. AMBJøRN \\ The Niels Bohr Institute, Copenhagen University \\ Blegdamsvej 17, 2100 Copenhagen Ø, Denmark \\ and \\ Institute for Mathematics, Astrophysics and Particle Physics (IMAPP) \\ Radbaud University Nijmegen \\ Heyendaalseweg 135, 6525 AJ, Nijmegen, The Netherlands \\ Y. WATABIKI \\ Tokyo Institute of Technology \\ Department of Physics, High Energy Theory Group \\ 2-12-1 Oh-okayama, Meguro-ku, Tokyo 152-8551, Japan
}

(Received April 10, 2017)

We describe a CDT-like model where breaking of $W^{(3)}$ symmetry will lead to the emergence of time and, subsequently, of space. Surprisingly, the simplest such models which lead to higher dimensional spacetimes are based on the four "magical" Jordan algebras of $3 \times 3$ Hermitian matrices with real, complex, quaternion and octonion entries, respectively. The simplest symmetry breaking leads to universes with spacetime dimensions $3,4,6$, and 10 .

DOI:10.5506/APhysPolBSupp.10.299

\section{Introduction}

String field theory is notoriously complicated, but the so-called noncritical string field theory $[1-3]$ is a lot simpler. An even simpler version is the so-called CDT string field theory [4]. The starting point is the continuum limit of two-dimensional causal dynamical triangulations (CDT) [5], a limit which is two-dimensional Hořava-Lifshitz quantum gravity if spacetime topology is trivial [6]. However, here we are interested in a generalized CDT where spacetime topology can change [7]. This theory describes the

* Talk presented by J. Ambjørn at the $3^{\text {rd }}$ Conference of the Polish Society on Relativity, Kraków, Poland, September 25-29, 2016. 
dynamics of topology changes of two-dimensional spacetime. One has creation and annihilation operators, $\Psi^{\dagger}(L)$ and $\Psi(L)$, for spatial universes of length $L$ and the Hamiltonian involves terms like

$$
\Psi^{\dagger}\left(L_{1}\right) \Psi^{\dagger}\left(L_{2}\right) \Psi\left(L_{1}+L_{2}\right), \quad \Psi^{\dagger}\left(L_{1}+L_{2}\right) \Psi\left(L_{2}\right) \Psi\left(L_{1}\right),
$$

which describe the splitting of a spatial universe in two, and the merging of two spatial universes into one such universe.

Although string field theory deals with splitting and merging of spatial universes, at the end of the day it tells us surprisingly little about the creation of a universe from "nothing", i.e. Big Bang. This led us to look for some general symmetry, the breaking of which could result in a Big Bang scenario. It is possible to derive the CDT string field Hamiltonian by starting out with a $W^{(3)}$-symmetric theory which a priori has no spacetime interpretation. However, when the $W^{(3)}$ symmetry is broken, "time" and correspondingly the (CDT) Hamiltonian will "emerge", but in such a way that also space can be created from "nothing". Below, we will shortly describe how this is realized. However, in this way, one only obtains a one-dimensional space. We will then argue that this one-dimensional scenario can be generalized to 2, 3, 5 and 9 spatial dimensions if one considers $W^{(3)}$ algebras with intrinsic symmetries related to one of the so-called "magical" Jordan algebras.

\section{Why $W^{(3)}$ ?}

When one discusses splitting and joining of strings, one encounters terms like (1). In the case of ordinary non-critical string field theory, this leads to a special $W^{(3)}$ algebra which ensures that the partition function is a $\tau$-function [8]. In the case of CDT string field theory, we now promote the $W^{(3)}$ symmetry to the starting principle.

The formal definition of $W^{(3)}$ operators in terms of operators $\alpha_{n}$ satisfying

$$
\left[\alpha_{m}, \alpha_{n}\right]=m \delta_{m+n 0}
$$

is the following:

$$
W_{n}^{(3)}=\frac{1}{3} \sum_{k, l, m}: \alpha_{k} \alpha_{l} \alpha_{m}: \delta_{k+l+m, n} .
$$

The normal ordering :(·): refers to the $\alpha_{n}$ operators $\left(\alpha_{n}\right.$ to the left of $\alpha_{m}$ for $n>m$. This ordering is opposite to the one conventionally used. See [3] for the motivation for such a choice.).

We then define the "absolute vacuum" $|0\rangle$ by the following condition:

$$
\alpha_{n}|0\rangle=0, \quad n<0,
$$


and the so-called $W$-Hamiltonian $\hat{H}_{W}$ by

$$
\hat{H}_{W}:=-W_{-2}^{(3)}=-\frac{1}{3} \sum_{k, l, m}: \alpha_{k} \alpha_{l} \alpha_{m}: \delta_{k+l+m,-2} .
$$

Note that $\hat{H}_{W}$ does not contain any coupling constants.

It was shown in [9] that by introducing a coherent state, which is an eigenstate of $\alpha_{-1}$ and $\alpha_{-3}$ and which we denoted the "physical" vacuum state $\mid$ vac $\rangle, \hat{H}_{W}$ was closely related to the CDT string field Hamiltonian $\hat{H}$. We thus defined

$$
|\operatorname{vac}\rangle \propto e^{\lambda_{1} \alpha_{1}+\lambda_{3} \alpha_{3}}|0\rangle
$$

and we have

$$
\alpha_{-1}|\operatorname{vac}\rangle=\lambda_{1}|\mathrm{vac}\rangle, \quad \alpha_{-3}|\operatorname{vac}\rangle=3 \lambda_{3}|\mathrm{vac}\rangle .
$$

The main point is the following: because $\left\langle\operatorname{vac}\left|\alpha_{n}\right|\right.$ vac $\rangle$ is different from zero for $n=-1$ and $n=-3, \hat{H}_{W}$ will now contain terms only involving two operators $\alpha_{l}$. These terms can act like quadratic terms in $\hat{H}$. At the same time, the cubic terms left in $\hat{H}_{W}$ will act like the interaction terms in $\hat{H}$, resulting in joining and splitting of universes. Finally, the expectation values of $\alpha_{-1}$ and $\alpha_{-3}$ determine the coupling constants of $\hat{H}$. More precisely, one has [9]

$$
\hat{H}_{W} \propto \hat{H}+c_{4} \phi_{4}^{\dagger}+c_{2} \phi_{2}^{\dagger},
$$

where $\hat{H}$ is the CDT string field Hamiltonian. $c_{4}$ and $c_{2}$ are constants. The creation operators $\phi_{n}^{\dagger}$ are the $\alpha_{n}, n>0$, while annihilation operators $\phi_{n}$ are related to $\alpha_{n}, n<0$, except that $\phi_{1}$ and $\phi_{3}$ are shifted by eigenvalues given in Eq. (7), such that $\phi_{n}|\operatorname{vac}\rangle=0 . \hat{H}$ is normal ordered such that $\hat{H}|\operatorname{vac}\rangle=0$.

By breaking the $W^{(3)}$ symmetry, one can thus obtain CDT string field theory except for one important point: the vacuum is not stable. The terms $c_{4} \phi_{4}^{\dagger}+c_{2} \phi_{2}^{\dagger}$ cause universes of infinitesimal length to be created and the non-interacting part of $\hat{H}$, which explicitly can be written as

$$
\hat{H}_{0}=-\sum_{l=1}^{\infty} \phi_{l+1}^{\dagger} l \phi_{l}+\mu \sum_{l=2}^{\infty} \phi_{l-1}^{\dagger} l \phi_{l},
$$

might expand such an infinitesimal length space to macroscopic size ${ }^{1}$.

1 The relation between the operators $\phi_{l}, \phi_{l}^{\dagger}$ and the operators $\Psi(L), \Psi^{\dagger}(L)$ which annihilate and create spatial universes of macroscopic length $L$ is as follows

$$
\Psi^{\dagger}(L)=\sum_{l=0}^{\infty} \frac{L^{l}}{l !} \phi_{l}^{\dagger}
$$




\section{Generalization to higher dimensions}

Above the spatial universe created from nothing was one-dimensional. We can introduce higher dimensional spaces by attaching intrinsic "flavors" to different spatial directions. In addition, we want to be able to rotate these flavors into each other. This leads to so-called extended $W^{(3)}$ algebras, which again are related to Jordan algebras [10]. Surprisingly, it turns out that only the four so-called magical Jordan algebras allow us to make symmetry breakings which lead to CDT-like Hamiltonians of the kind considered above.

We find for the $W^{(3)}$ Hamiltonian the expression

$$
\hat{H}_{\mathrm{W}}:=-W_{-2}^{(3)}=-\frac{1}{3} \sum_{k, l, m} \sum_{a, b, c} d_{a b c}: \alpha_{k}^{(a)} \alpha_{l}^{(b)} \alpha_{m}^{(c)}: \delta_{k+l+m,-2},
$$

where $d_{a b c}$ are the structure constants for the Jordan algebras. The magical Jordan algebras are Hermitean $3 \times 3$ matrices $H_{3}(\mathbb{F})$, where $\mathbb{F}$ denotes $\mathbb{R}, \mathbb{C}, \mathbb{H}$ and $\mathbb{D}$ (the real numbers, the complex number, the quaternions and the octonions), and the structure constants are related to the standard Gell-Mann $d_{a b c}$ for $H_{3}(\mathbb{C})$ in a simple way. Again, the model only allows a spacetime interpretation after choosing a specific coherent state. Here, we discuss only the simplest, interesting choice, namely breaking in the 8-direction (in the notation of Gell-Mann). Instead of (6) and (7), we can choose

$$
|\mathrm{vac}\rangle_{8} \propto e^{\lambda_{1}^{(8)} \alpha_{1}^{(8)}+\lambda_{3}^{(8)} \alpha_{3}^{(8)}}|0\rangle,
$$

and we have

$$
\alpha_{-1}^{(8)}|\mathrm{vac}\rangle_{8}=\lambda_{1}^{(8)}|\mathrm{vac}\rangle_{8}, \quad \alpha_{-3}^{(8)}|\mathrm{vac}\rangle_{8}=3 \lambda_{3}^{(8)}|\mathrm{vac}\rangle_{8} .
$$

Again, one obtains an unstable vacuum. The kinetic, non-interacting part of the Hamiltonian (the part corresponding to (9)) has coefficients $d_{a b 8}$ and, in fact, only coefficients $d_{a a 8}$ are different from zero. One can argue [11] that in order for the non-interacting part of the Hamiltonian to allow a universe to expand from infinitesimal size to macropscopic size, one has to demand that the coefficients $d_{a a 8}>0$. When we then look at the four magical algebras, we have for $H_{3}(\mathbb{R})$ two indices $a$, where $d_{a a 8}=1 / \sqrt{3}$. For $H_{3}(\mathbb{C})$, we have three indices $a$, where $d_{a a 8}=1 / \sqrt{3}$. For $H_{3}(\mathbb{H})$, we have five indices $a$, where $d_{a a 8}=1 / \sqrt{3}$ and, finally, for $H_{3}(\mathbb{D})$, we have nine indices $a$, where $d_{a a 8}=1 / \sqrt{3}$. The rest of the $d_{a a 8}$ are non-positive. The symmetry breaking corresponds to breaking the automorphism group of the $W^{(3)}$ algebras from $\mathrm{SO}(3)$ to $\mathrm{SO}(2)$, from $\mathrm{SU}(3)$ to $\mathrm{SO}(3)$, from $\mathrm{USp}(6)$ to $\mathrm{SO}(5)$ and, finally, from $F_{4}$ to $\mathrm{SO}(9)$. The extended spacetime dimensions will be (including the time) $2+1,3+1,5+1$ and $9+1$ which are the dimensions of the classical superstrings. It is an interesting question if one can consider the flavor indices $a$ of space as reflecting matter fields partly 
integrated out. It opens for the exciting possibility that the matter content is unique.

J.A. and Y.W. acknowledge support from the ERC-Advance grant 291092, "Exploring the Quantum Universe" (EQU).

\section{REFERENCES}

[1] N. Ishibashi, H. Kawai, Phys. Lett. B 314, 190 (1993) [arXiv:hep-th/9307045]; 322, 67 (1994) [arXiv:hep-th/9312047]; 352 , 75 (1995) [arXiv:hep-th/9503134].

[2] Y. Watabiki, Nucl. Phys. B 441, 119 (1995) [arXiv:hep-th/9401096]; Phys. Lett. B 346, 46 (1995) [arXiv:hep-th/9407058].

[3] J. Ambjørn, Y. Watabiki, Int. J. Mod. Phys. A 12, 4257 (1997) [arXiv:hep-th/9604067].

[4] J. Ambjørn et al., J. High Energy Phys. 0805, 032 (2008) [arXiv:0802.0719 [hep-th]].

[5] J. Ambjørn, R. Loll, Nucl. Phys. B 536, 407 (1998) [arXiv:hep-th/9805108].

[6] J. Ambjørn, L. Glaser, Y. Sato, Y. Watabiki, Phys. Lett. B 722, 172 (2013) [arXiv:1302.6359 [hep-th]].

[7] J. Ambjørn, R. Loll, W. Westra, S. Zohren, J. High Energy Phys. 0712, 017 (2007) [arXiv:0709.2784 [gr-qc]]; J. Ambjørn et al., Phys. Lett. B 665, 252 (2008) [arXiv:0804.0252 [hep-th]]; J. Ambjørn, T.G. Budd, J. Phys. A: Math. Theor. 46, 315201 (2013) [arXiv:1302.1763 [hep-th]].

[8] M. Fukuma, H. Kawai, R. Nakayama, Int. J. Mod. Phys. A 6, 1385 (1991); Commun. Math. Phys. 143, 371 (1992); A. Mironov, A. Morozov, Phys. Lett. B 252, 47 (1990); A. Marshakov, A. Mironov, A. Morozov, Mod. Phys. Lett. A 7, 1345 (1992).

[9] J. Ambjørn, Y. Watabiki, Phys. Lett. B 749, 149 (2015) [arXiv:1505.04353 [hep-th]].

[10] L.J. Romans, Nucl. Phys. B 352, 829 (1991).

[11] J. Ambjørn, Y. Watabiki, arXiv:1703.04402 [hep-th]. 